\title{
OSCE Virtual: Simulação de Avaliação de Casos Clínicos
}

\author{
Adja Ferreira de Andrade', Charles Andryê G. Madeira', Samanta Ferreira Aires ${ }^{1}$ \\ ${ }^{1}$ Instituto Metrópole Digital - Universidade Federal do Rio Grande do Norte (UFRN) \\ Caixa Postal 15.064 - 91501-970 - Natal - RN - Brasil \\ \{adja, charles\}@imd.ufrn.br, samantabti@gmail.com
}

\begin{abstract}
This article describes a new approach for the simulated evaluation of the medicine, called OSCE (Objective Structured Clinical Examination), which is also known in the literature as clinical examination structured by stations. The main goal in here is to create a virtual environment that allows to train students with this evaluation model. The Virtual OSCE consists in simulating clinical care for evaluating student behaviors, mastery and skills while performing tasks in order to solve several clinical cases.
\end{abstract}

Resumo. O presente artigo propõe uma nova abordagem para a avaliação simulada da medicina, chamada OSCE (Objective Structured Clinical Examination), também conhecida na literatura médica como exame clínico estruturado por estações. O objetivo principal da presente proposta é criar um ambiente virtual para permitir o treinamento de estudantes com este modelo de avaliação. A OSCE Virtual consiste na simulação de um atendimento clínico no qual são avaliados comportamento, domínio e habilidades dos estudantes durante a execução das tarefas necessárias para a resolução de diversos casos clínicos.

\section{Introdução}

A avaliação simulada da medicina, chamada OSCE (Objective Structured Clinical Examination), também conhecida como exame clínico estruturado por estações, é um modelo de avaliação simulada que consiste na realização de tarefas para análises clínicas usando várias estações nas quais cada uma representa um caso clínico [Turner and Dankoski 2008]. Esse método de avaliação de habilidades clínicas foi desenvolvido há mais de 20 anos, na Escócia, e tem ganhado crescente aceitação e difusão por todo o mundo [Troncon 2007]. Em cada estação, os alunos examinados são solicitados a desempenhar tarefas clínicas distintas, como obter uma história clínica focalizada, realizar o exame de um órgão, inspecionar uma radiografia, analisar um traçado eletrocardiográfico ou instruir um paciente sobre o seu diagnóstico.

Durante a avaliação, os alunos permanecem em cada estação por um tempo predeterminado, onde realizam a tarefa solicitada sob a supervisão de um avaliador, empregando um instrumento de registro pré-elaborado. Nesse registro, há um protocolo de observação, contendo as ações que os professores esperam que sejam efetuadas pelos alunos. A realização dessas ações implica na demonstração do domínio das habilidades dos alunos avaliados em exame. Ao término do tempo previsto, quando se emite um sinal sonoro audível por todos, os estudantes passam para a estação seguinte, alternando 
a ocupação das várias estações. A Figura 1 mostra um exemplo de exame em uma estação onde encontram-se presentes um paciente (ator), um aluno sendo avaliado e um avaliador (professor).

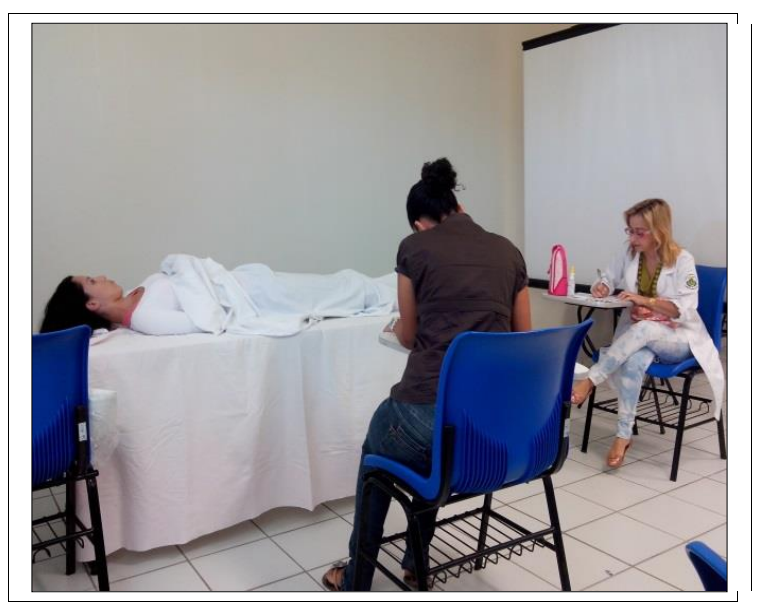

Figura 1. Cenário de avaliação da OSCE.

Este artigo propõe a construção de um ambiente virtual para simulação e treinamento de estudantes no contexto da avaliação OSCE, representando um atendimento médico de consultório em que o usuário deve realizar a avaliação diagnóstica através de diálogos e exames. A OSCE Virtual se insere dentro de um ambiente hospitalar, sendo composta basicamente de dois cenários principais: corredores de um hospital que levarão os usuários às diferentes estações existentes, e as próprias estações (ou salas) configuradas com os casos clínicos a serem tratados.

\section{Avaliação Diagnóstica}

No contexto educacional, a avaliação implica em se obter informações do desempenho do aluno por meio da aplicação de métodos específicos, os quais podem subsidiar a tomada de decisão no processo de ensino, os objetivos instrucionais previamente estabelecidos, e a mediação do professor. O processo de avaliação permite, também, verificar se o estudante está em condições de progredir nas etapas futuras de sua formação. Adicionalmente, a aplicação dos diferentes instrumentos de avaliação fornece resultados que possibilitam diferenciar os estudantes em diversos graus de conhecimento. Há uma tendência negativa quanto ao uso da avaliação predominantemente para se medir a aquisição de conhecimento e não para apreciação de habilidades e de competências mais abrangentes e de ordem mais prática.

Dentre os vários modelos de avaliação, temos a avaliação formativa, que é aquela realizada, regular e periodicamente, ao longo do processo educacional. Nesta forma de avaliação, obtém dados sobre o progresso conseguido e, desse modo, efetiva-se a oportuna correção das distorções observadas, preenchendo as lacunas detectadas, bem como reforçando as conquistas realizadas. Segundo Troncon [Troncon 2007], uma importante característica desse tipo de avaliação é o feedback imediato que deve ser fornecido ao estudante, de modo a configurar o processo de obtenção de dados como genuína atividade educacional. 
A avaliação diagnóstica pode ser empregada para a detecção da ocorrência de problemas ou dificuldades específicas de aprendizado, a confirmação e caracterização desses problemas, de modo a se dispor de dados para a sua solução.

Algumas propostas utilizam a abordagem de simulação como metodologia educativa [Camilo 2008; Courteille 2008; Troncon 2007; Lok 2006]. Na proposta de [Camilo 2008] a abordagem é centrada no aluno e nas suas necessidades de aprendizagem, ao invés de se centrar no doente, como acontece na maior parte das vezes em contexto clínico. Segundo o autor, tal abordagem "proporciona uma exposição pró-ativa e controlada dos alunos a desafios clínicos progressivamente mais complexos, incluindo aquelas situações potencialmente fatais, que não poderiam ser treinados doutra forma". A aprendizagem através da simulação é eficaz. A adaptabilidade e versatilidade dos simuladores proporcionam múltiplas experiências de aprendizagem, a aprendizagem individualizada e ativa dos alunos que assim não se apresentam como meros espectadores, a aprendizagem sistemática de competências na comunicação e na atitude e de trabalho em equipe dificilmente realizáveis noutros contextos.

O ambiente de simulação [Courteille 2008] tem como objetivo avaliar as competências e habilidades para resolver um caso com pacientes virtuais (VP), que inclui anamnese, exames laboratoriais, exames físicos e sugestão de um diagnóstico preliminar dos alunos. O objetivo principal deste estudo é avaliar o potencial de um VP como uma possível ferramenta para a avaliação do raciocínio clínico e habilidade de resolver problemas entre os estudantes de medicina. A sensação de realismo do VP e seu possível impacto afetivo na confiança do estudante também foram investigados. Neste estudo observou-se e analisou as reações dos alunos, engajamento e performance (arquivos de log de atividades) durante as sessões interativas com a simulação.

Já existem projetos com a mesma proposta do Osce Virtual, como por exemplo o sistema denominado VOSCE (Virtual OSCE) desenvolvido por Benjamin Lok [Lok 2006] e sua equipe. É um sistema que utiliza personagens virtuais para ajudar na construção das habilidades de comunicação médico-paciente. $\mathrm{O}$ ambiente permite que os estudantes de medicina possam entrevistar uma paciente Virtual DIANA (Digital Animated Avatar) usando discurso e gestos. Um instrutor virtual, VIC (Virtual Interactive Character), fornece um feedback imediato sobre o desempenho do aluno. Podemos perceber que esse ambiente, apesar de possuir proposta semelhante à nossa, tem o processo de execução diferente, pois ele é mais focado na entrevista médicopaciente, realizado através da projeção de uma imagem de consultório na parede, assim os estudantes recebem um tablet com a tarefa, e executam o atendimento através da conversação com Diana. Os movimentos dos alunos, tais como gestos de apontar para determinada parte do corpo de Diana são detectados por câmeras instaladas, e há o reconhecimento de voz, para que eles possam fazer perguntas à Diana. A paciente é programada com as possíveis respostas; o instrutor vai dando as direções que os estudantes devem tomar, mostrando em que eles estão errando. O ambiente VOSCE foi desenvolvido com apenas um caso de dores abdominais agudas.

Um outro software já desenvolvido é o Paciente Virtual: simulador de casos. O ambiente já está em uso pelos estudantes da Fundação Santa Fé de Bogotá [SPV 2013], trata-se de um aplicativo web e tem a funcionalidade bem parecida com a nossa. Nele, os 
estudantes devem entrar no sistema através de um login, e dentro do sistema se deparam com um menu com as opções de solucionar um caso, visualizar casos resolvidos, editar suas informações pessoais e mudar a senha. Quando o aluno seleciona um novo caso, ele é levado para uma interface com várias abas: Apresentação, Identificação, Interrogatório, Exame Físico, Diagnóstico, entre outros. Uma aba interessante é a de Interrogatório, onde as perguntas são feitas através de palavras chaves, isto é, o estudante digita uma palavra e assim surgem várias perguntas possíveis relacionadas àquela palavra, e o sistema já possui um banco de respostas. Uma peculiaridade é que o exame físico está restrito às áreas do corpo relacionadas a cada doença; já a nossa o aluno é livre para examinar todas as áreas do corpo do paciente.

A proposta deste trabalho se aproxima de alguma das abordagens acima mencionadas. A OSCE Virtual é baseada no modelo de avaliação diagnóstica. Tal abordagem consiste na simulação dos comportamentos do estudante, que indicam seu domínio e aptidão durante a execução de tarefas estabelecidas pelos avaliadores. O modelo busca categorizar a competência médica em vários domínios como, por exemplo, o conhecimento médico, assistência ao paciente, profissionalismo, comunicação, habilidades interpessoais, entre outros.

No processo de avaliação tradicional da OSCE [Newble 2004] estão envolvidos os seguintes atores: o avaliador, o avaliado e o paciente. Cabe ressaltar que a atuação do avaliador é feita através de um protocolo de observação pré-estabelecido. Dessa forma, todos os alunos são avaliados exatamente nas mesmas condições. Essas são as características que permitem preencher, com mais facilidade, os requisitos de fidedignidade da avaliação e conferem objetividade ao exame.

É neste protocolo onde estão descritas todas as ações que devem ser realizadas pelos alunos. Estas informações são vistas apenas pelo avaliador, que é quem registra e avalia o comportamento dos alunos durante a tarefa. Cada ação listada possui uma pontuação e representa um quesito tal qual os descritos a seguir:

- Apresentar-se e cumprimentar o paciente (0,5 pontos);

- Pesquisar adequadamente os sinais referentes à tarefa. Por exemplo, se uma questão pedir ao estudante para analisar os sintomas de meningite, o aluno deverá executar os passos necessários para detectar se o paciente possui essa doença. Fazendo os exames de Rigidez da Nuca, Brudzinski (levantamento involuntário das pernas), Laségue (levantamento do membro inferior com os joelhos estendidos) e outros exames específicos para a tarefa. Para cada exame efetuado de forma correta, o aluno recebe uma pontuação (em média 1,0 ponto);

- Explicar ao paciente adequadamente sobre a doença (1,0 ponto);

- Explicar o tratamento a ser efetuado para a cura/melhora do paciente $(1,0$ ponto);

- Quesitos específicos das doenças a serem pesquisadas.

Com a finalidade de atingir o objetivo do processo de diagnóstico, o aluno pode efetuar várias ações tais como examinar o paciente fisicamente, analisar o seu histórico e solicitar exames laboratoriais para obter mais informações sobre o seu caso clínico, confirmar suspeitas e validar hipóteses iniciais. O objetivo é fornecer uma diretriz inicial 
de análise do caminho a ser percorrido no atendimento para, finalmente, poder dar um diagnóstico e prescrever um tratamento para o caso em questão. Também é dada ao aluno a possibilidade de diagnosticar o caso antes mesmo de efetuar qualquer ação.

A opção de diagnosticar deve estar visível durante o atendimento. Quando o aluno der um diagnóstico, por se tratar do propósito da avaliação, esse será encaminhado para o professor, que corrigirá e dará um retorno ao aluno com suas considerações e correções. Os critérios levados em consideração são os seguintes: assistência ao paciente, pesquisa dos sinais específicos de cada doença, comunicação interpessoal, dentre outros.

\section{Metodologia}

A metodologia empregada para o desenvolvimento do projeto da OSCE Virtual foi iniciada com um levantamento dos requisitos da simulação através da realização de diversas reuniões com professores da área da saúde responsáveis pela elaboração e aplicação das avaliações de casos clínicos. Nesta fase, foram estudadas as necessidades para a construção de um protótipo inicial do simulador, considerando para isto o contexto de alguns casos selecionados para estudo.

Para formalizar este processo, uma tarefa conhecida como game design [Shell 2008; Schuytema 2008] se mostrou adaptada para permitir a criação de uma simulação baseada no formato de um jogo digital, onde aspectos lúdicos também se fazem presentes. Este processo de formalização consistiu na concepção de roteiro, script, descrição de personagens, além de uma série de atividades descrevendo os elementos da simulação. $O$ principal objetivo deste processo é a construção de um documento de diretrizes no qual são considerados quatro elementos principais do programa - estória, estética, mecânica e tecnologia - conforme descritos a seguir no contexto da OSCE Virtual.

\subsection{Estória}

A proposta da OSCE Virtual consiste em simular atendimentos médicos. Para o nosso primeiro protótipo, decidimos simplificar esse contexto tomando como referência a descrição de apenas algumas configurações de estações como, por exemplo, o modelo de caso de meningite. Neste caso de estudo, o cenário da estação consiste em uma sala onde um paciente encontra-se deitado num leito de hospital, com o corpo totalmente visível para que o estudante possa examiná-lo da melhor maneira que the convenha.

Durante o exame, o aluno pode executar diversas ações como ver o histórico do paciente (nome, idade, estado civil, naturalidade), requisitar exames, analisar sintomas, avaliar o seu estado físico e mental, suas internações, doenças preexistentes, dentre outras informações. Baseando-se nos dados e sintomas, o aluno pode refletir sobre o diagnóstico de qual a possível doença que o paciente possui e como ela deve ser tratada. Estas ações, que são realizadas durante o atendimento, visam simular o mais próximo possível o que acontece em um consultório real a fim de treinar o aluno da melhor forma.

\subsection{Estética: A Interface do Ambiente}

No simulador da OSCE Virtual, temos o aluno e o professor como atores primários e um monitor e um administrador como atores secundários. $\mathrm{O}$ paciente da avaliação existe apenas como um objeto de interface. 
A interface do jogo baseia-se na funcionalidade de um hospital real. Ao entrar no simulador, o usuário é direcionado para uma tela principal que apresenta um corredor de hospital. Neste corredor (ver Figura 2a) poderão aparecer diversas portas, macas e também pessoas, cada uma das portas representando uma estação onde um caso préconfigurado existe para um paciente. O usuário poderá caminhar pelo corredor e escolher qual porta deseja entrar.

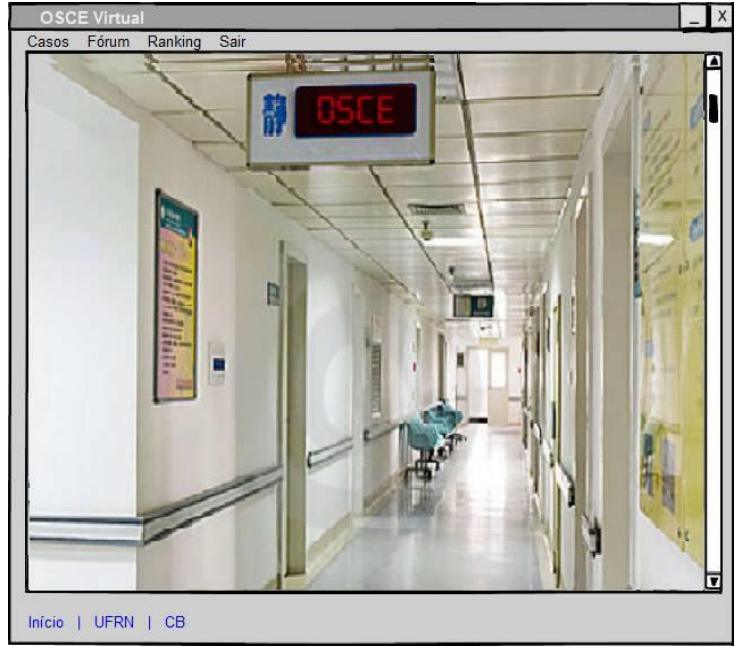

a)

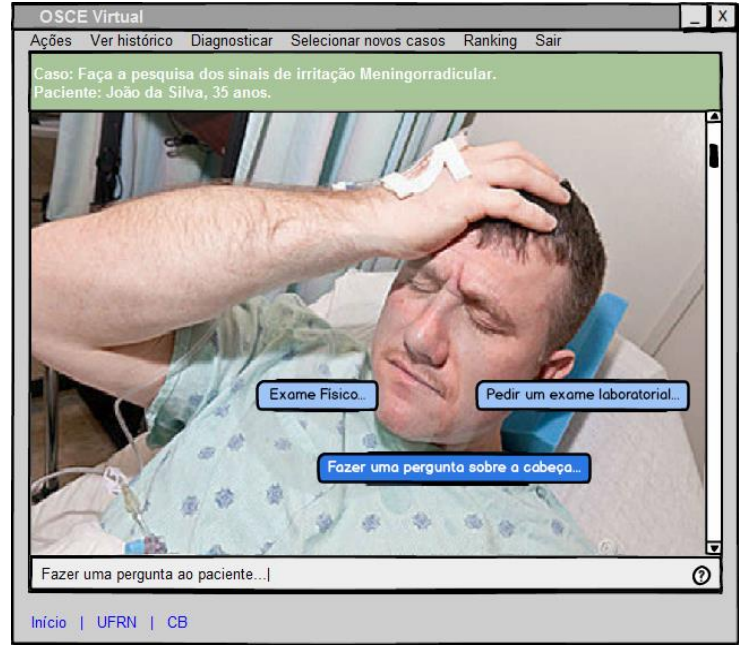

b)

Figura 2. a) Interface de acesso aos casos configurados para as estações existentes; b) Cenário de interação do estudante com um paciente

Uma vez uma das portas selecionada, o usuário se depara com uma sala de atendimento onde um paciente espera para ser atendido e ter seu caso diagnosticado. Entrando nesta sala, um novo cenário que permite interação com o paciente é apresentado (ver Figura 2b) e disponibiliza diversas informações tais como a tarefa a ser executada, o título do caso, nome, idade e histórico do paciente, ações possíveis de diagnóstico, selecionar novos casos, etc. No exemplo da figura acima temos os dados para o seguinte caso:

- Caso: faça a pesquisa dos sinais de irritação meningorradicular;

- Paciente: João da Silva, 35 anos.

Para iniciar o processo de examinação do paciente, o usuário deve guiar o simulador com as suas ações uma vez que esta opção não é apresentada de forma explícita na interface gráfica. Para que esta opção se torne visível, o usuário deve mover o cursor do mouse sobre qualquer parte do corpo do paciente para buscar as opções existentes no cenário. Este processo é descrito na seção 3.3.

O diagnóstico pode ser efetuado através do menu Diagnosticar que direcionará o usuário para uma caixa de texto, onde ele deverá redigir as suas conclusões baseadas nos exames feitos com o paciente, a constatação de uma possível doença e qual tratamento deve ser prescrito para o paciente. O diagnóstico dado é então enviado ao professor. Em seguida, outros casos poderão ser selecionados para iniciar novamente o processo de atendimento.

No menu da interface gráfica são também apresentadas opções para participação em 
fóruns e consulta ao ranking de alunos. Nos fóruns, os estudantes podem postar tópicos sobre assuntos relacionados aos diversos casos e enviar dúvidas ao professor. Esses tópicos ficarão visíveis para todos os alunos e professores cadastrados no sistema, onde todos poderão responder às questões e discutirem sobre os casos. Quanto ao ranking, este será usado como forma de estímulo para os alunos. Ele apresentará uma classificação dos usuários do simulador de acordo com a pontuação obtida através da resolução dos diferentes casos. A pontuação do simulador é definida de acordo com as expectativas do protocolo de atendimento. Assim, os estudantes que conseguirem diagnosticar doenças seguindo todos os pontos requeridos pelo protocolo, ou que realizem exames em menos tempo, ou então que acertem realmente a doença a ser tratada, obterão um número maior de pontos e se classificarão entre os primeiros do ranking.

\subsection{Mecânica}

A mecânica do simulador é inspirada nas interações do jogo The Sims [EA Games 2013]. Este jogo retrata uma simulação da vida real, em que o usuário pode criar um ou vários personagens, construir casas e até mesmo cidades inteiras. As interações entre personagens do The Sims são feitas de forma que o diálogo é baseado em um processo de escolha de ação seguida de reação do interlocutor. As ações podem ser simples ou compostas, sendo o próprio usuário o único guia no processo de interação.

Tomando como referência a mecânica do The Sims, a interação na OSCE Virtual é baseada nas opções de escolhas e diálogos, as ações sendo efetuadas através da movimentação do mouse e clique de seus botões que permite abrir opções de interação através de janelas de pop-up.

Quando o usuário do simulador escolhe examinar o paciente e seleciona uma região do corpo que lhe convém para ser examinada, surgem então as opções existentes para esta região. A partir deste ponto o usuário pode então começar a interagir com o paciente. Por exemplo, se ele desejar questionar o paciente sobre os sintomas aparentes na sua cabeça, então poderá mover o cursor do mouse sobre esta região do corpo onde haverá um aviso de que a região pode ser examinada. Este aviso pode ser emitido mudando a cor da cabeça, tornando-a mais clara ou algo do gênero. A partir de um clique do botão nesta região, surgirá uma janela de pop-up com várias perguntas possíveis sobre o órgão selecionado, tais como: "sua cabeça dói?", "o senhor bateu a cabeça em algum lugar?", "o senhor apresenta tontura?". O sistema dispõe também de outras ações que podem ser efetuadas na região examinada, como por exemplo apalpar, mover e levantar, e para cada uma dessas ações existe uma resposta do paciente, dizendo se a ação teve algum efeito ou não.

O fato do simulador não apresentar, de forma explícita, a opção de examinação do paciente permite deixar o sistema com uma postura imparcial, sendo o usuário o seu único guia nas interações que o levarão a dar um diagnóstico para o caso em questão. Desta forma, fazemos com que o nosso modelo de processo de avaliação virtual se torne mais próximo do modelo de avaliação real da OSCE, onde o avaliado não é guiado por nenhum sistema ou pessoa, além do paciente, durante o processo de examinação e diagnóstico.

A representação formal do modelo é feita através de casos de uso. Um caso de uso serve 
para comunicar requisitos entre usuários e desenvolvedores, modelando os atores e os papéis que eles executam ao interagir com o sistema [Rosenberg e Stephens 2007]. Cada caso de uso consiste em um completo curso de eventos do sistema, iniciado por um ator. $\mathrm{Na}$ figura 3 exemplificamos, através de um diagrama de sequência, como é dada essa interação no caso da meningite:

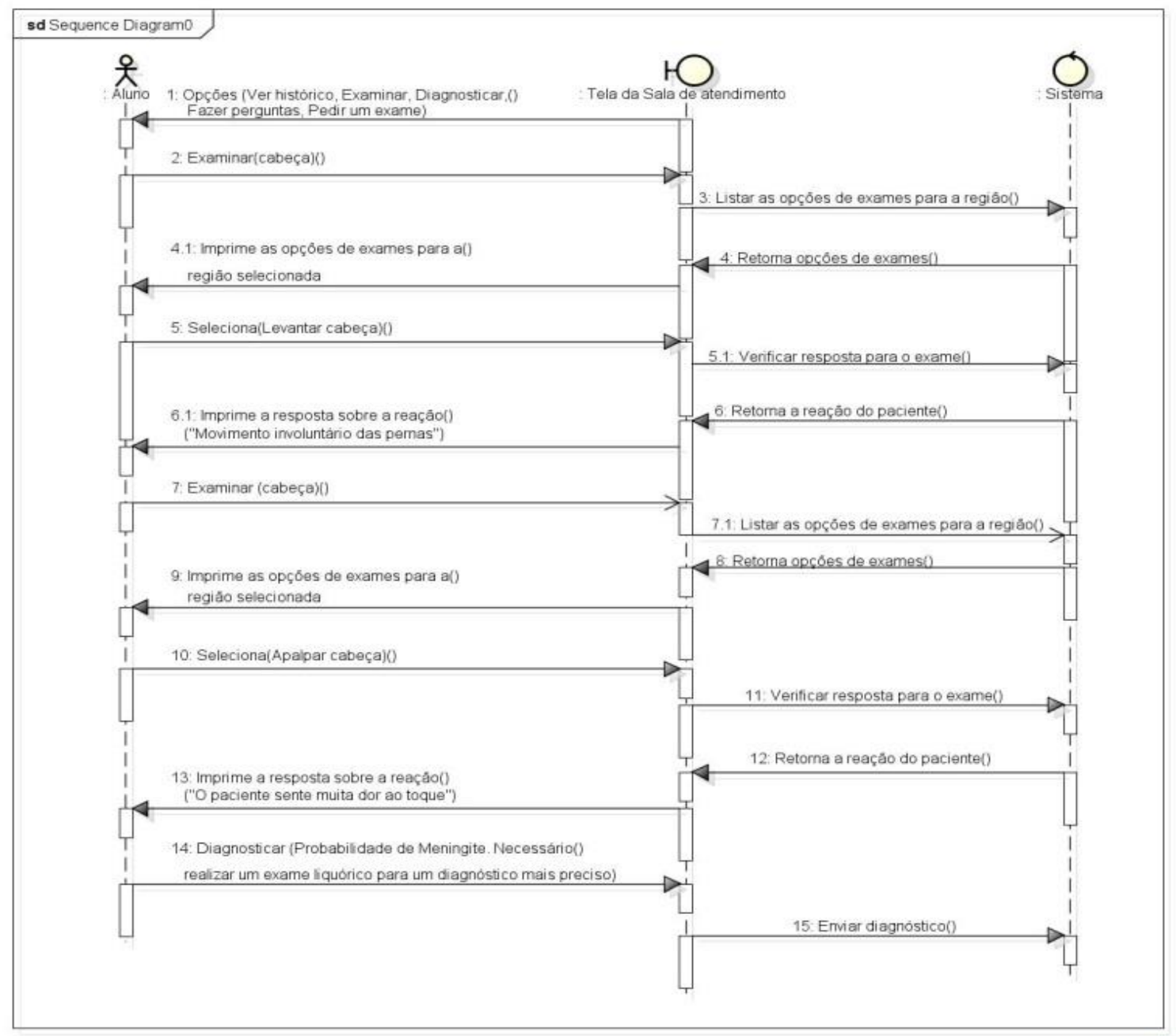

Figura 3. Diagrama de sequência do Caso Meningite

Durante o exame clínico, o usuário do simulador é livre para realizar qualquer ação que desejar. Assim, se o paciente estiver, por exemplo, reclamando de fortes dores de cabeça, e o aluno for examinar o pé do paciente, isto poderá eventualmente acarretar em perda de pontos na sua avaliação. Esse é o tipo de liberdade de escolha que o aluno terá na OSCE Virtual devido às diversas opções de ações existentes para as diferentes regiões selecionadas. O sistema define apenas quais as áreas do corpo que poderão ser examinadas, permitindo tornar o simulador mais divertido e didático para o aluno.

\subsection{Tecnologia}

Para a implementação do protótipo do ambiente, ficou clara a necessidade de que o sistema deveria permitir ao aluno um acesso contínuo ao ambiente virtual para poder efetuar o treinamento necessário de acordo com as suas necessidades, o que consistia em 
propor um simulador de ambiente hospitalar online e multiplataforma de fácil acesso através de smartphones e tablets. Para isto, escolhemos a linguagem HTML5 [Seidelin 2011; Geary 2012] como tecnologia a ser usada, devido a sua portabilidade, facilidade de uso e crescente espaço que tem ganhado recentemente no mercado de simulações e jogos digitais.

Para evitar o desenvolvimento de código de baixo nível, como tratamento de renderização, desenho de primitivas, tratamento de streams de entrada e saída, etc. e consequentemente acelerar a produção do simulador, motores de jogos são geralmente usados durante a fase de desenvolvimento. Os motores de jogos [Gregory et al. 2009] são modularizados para tratar de funções específicas no ciclo de desenvolvimento dos jogos digitais. Existe, por exemplo, motores gráficos responsáveis por toda a camada gráfica de um jogo, como cenários e texturas, facilitando a programação e a simulação de fenômenos reais. Essa modularização permite uma melhor separação entre a parte artística e a parte de lógica da programação.

Portanto, decidimos por fazer um estudo sobre diversos motores existentes na atualidade, específicos para o desenvolvimento de jogos digitais em HTML5, a fim de escolher um dentre eles que fosse mais adaptado ao nosso caso. Dentre os motores pesquisados, escolhemos o Crafty Engine [Crafty 2013], dado sua arquitetura simples e eficaz baseada em componentes, seu código aberto de tamanho reduzido e de fácil leitura, uma grande disponibilidade de tutoriais e exemplos a ela voltados, e a independência em relação às plataformas.

\section{Desafios}

Cabe ressaltar que embora a ênfase atual do projeto seja dada à simulação de alguns casos clínicos já delimitados pela equipe de Biociências, esparamos que este ambiente torne-se uma plataforma de autoria para inserção de novos casos-clínicos pelos professores. Este modelo está sendo desenvolvido no formato de formulário contendo diversas informações tais como: nome do novo caso, definição da tarefa a ser realizada, imagens e histórico médico do paciente, ações que devem ser efetuadas pelos alunos incluindo os tipos de interação para cada uma das ações. A ideia é que o simulador possa permitir a inserção de casos mais complexos englobando vários conceitos clínicos, garantindo ao ambiente um maior ciclo de vida.

Outro desafio é determinar qual o objetivo real da nossa avaliação. Uma hipótese que trabalhamos até o momento é que a simulação servirá para o treinamento dos alunos através de uma ferramenta computacional. Mas poderemos mais na frente também pensar na possibilidade do simulador dar suporte ao próprio processo de avaliação tradicional da OSCE.

Também é necessário estabelecer como será o processo avaliativo do diagnóstico realizado pelo aluno. Se apenas seguiremos com a proposta de enviar ao professor o diagnóstico dado pelo aluno, e este por sua vez se encarregará de dar um retorno avaliativo, ou se também poderíamos pensar em dar o retorno através do próprio sistema, onde uma espécie de sistema inteligente simulando o processo avaliativo poderia ser desenvolvido e colocado em prática. 


\section{Conclusões}

Neste trabalho, apresentamos uma proposta de simulador virtual para atendimento médico. $\mathrm{O}$ ambiente do simulador permite interagir com um paciente, efetuar ações e diagnosticar sobre os casos clínicos encontrados. O professor recebe tal diagnóstico e utiliza um protocolo de avaliação para analisá-lo. Este protocolo trata desde o até a maneira relacional de tratamento do paciente até o seu desempenho na atividade de diagnosticar casos clínicos. Esse modelo de avaliação é bastante importante, na medida em que constitui um poderoso instrumento de inspeção de como os alunos de medicina estudam e aprendem.

A simulação como metodologia avaliativa tem muitas vantagens tanto para o aluno como para os professores. Por exemplo, a OSCE Virtual promove um aprendizado seguro e a experiência com variedades de condições que são pouco frequentes na prática, pois na área da saúde é de extrema importância que os alunos pratiquem bastante, mas essa prática em um ambiente real é de difícil organização e de custo relativamente caro comparado com o ambiente virtual. Além disso, a simulação também permite uma avaliação em um ambiente realista, o que não distância os alunos da prática em um ambiente real. Com o uso de um ambiente virtual para a simulação de casos, os alunos poderão, de certa modo, praticar seus "erros" para que, quando foram avaliados de maneira real, esses erros sejam mínimos, tornando a prática mais segura para os futuros profissionais de medicina.

Do ponto de vista pedagógico, podemos ressaltar algumas vantagens como a formação para uma comunicação adequada com os pacientes, a avaliação do raciocínio clínico dos alunos na identificação e na solução do problema, o que leva em consideração a tomada de decisão médica, as reações dos alunos e dos pacientes. Esses são pontos importantes a serem avaliados dentro da OSCE Virtual.

A simulação de casos clínicos possui como vantagens o baixo custo, pois para uma simulação padronizada, isto é, uma simulação real, é necessária toda uma infraestrutura de apoio ao aluno, como por exemplo, a simulação da OSCE Real é feita dentro do Hospital Universitário, utiliza salas de atendimento, macas, cadeiras, e materiais necessários para a simulação. Normalmente, é agendado um dia para que todos os avaliadores, atores e alunos organizem os cenários dos casos a serem tratados, o que minimiza as quantidades de simulações praticadas durante o curso. No ambiente da OSCE Virtual a única coisa necessária é um computador com acesso à internet. Assim os alunos podem praticar no momento que lhes for conveniente, e também os professores podem analisar e dar o feedback no tempo que tiverem disponíveis. Essa é uma grande vantagem da utilização de simuladores.

Outra vantagem é a mobilidade, pois o atendimento dos casos pode ser acessado online através de várias plataformas como $\mathrm{PC}$, smartphones, tablets, etc. $\mathrm{O}$ ambiente encontrase atualmente na fase de design de interfaces, onde estão sendo especificados para a simulação elementos de pontuação e ranking, e interações com outros jogadores (através do fórum) garantindo uma experiência de troca de conhecimentos entre os alunos. A simulação visa usar cenários no estilo dos jogos digitais para chamar a atenção dos 
estudantes e tornar-se um recurso lúdico e didático a favor da educação, focado no processo de análise e reflexão.

\section{Referências}

Camilo, H. (2008). Educação Médica Baseada na Simulação e em Simuladores (2008).

Universidade de Coimbra. Fichas pedagógicas. Disponível em:

http://www.uc.pt/fmuc/gabineteeducacaomedica/fichaspedagogicas/Essencia_08.

Acessado em 05/10/2013.

Courteille, O. et al (2008). The use of a virtual patient case in an OSCE-based exam--a pilot study. Em MedTeach.Disponível em: http://www.ncbi.nlm.nih.gov/pubmed/18484444. Acessado em 05/10/2013.

Crafty (2013). Disponível em http://craftyjs.com/. Acessado em 04/10/2013.

EA Games (2013). The Sims. Disponível em http://www.thesims.com/pt-br. Acessado em $05 / 10 / 2013$.

Geary, D. (2012) Core HTML5 Canvas: Graphics, Animation, and Game Development. Prentice Hall.

Gregory, J., Lander, J. and Whiting, M. (2009) Game Engine Architecture. A K Peters/CRC Press.

Lok, B. et al. (2006). The use of virtual patients to teach medical students history taking and communication skills. In American Journal of surgery 191, 806-811.

Newble, D. I. (2004) Techniques for measuring clinical competence: objective structured clinical examinations. Med Educ. Cap. 38: 199-203.

Rosenberg, D. and Stephens, M. (2007) Use Cases Driven Object Modeling with UML Theory and Practice. Apress.

Schell, J. (2008) The Art of Game Design. Elsevier.

Schuytema, P. (2008) Design de Games: Uma Abordagem Prática. Cengage Learning.

Seidelin, J. (2011) HTML5 Games: Creating Fun with HTML5, CSS3, and WebGL. Wiley, 2011.

SPV (2013): Paciente Virtual: Simulador de Casos Clínicos. Fundação Santa Fé de Bogotá, Colômbia. Disponível em: http://fsfb.org.co/node/548. Acessado em $\underline{05 / 10 / 2013}$.

Troncon, L.E. de A.(2007) Utilização de Pacientes Simulados no Ensino e na avaliação de habilidades clínicas. Simpósio: Didática II - simulação, Vol. V, Ribeirão preto.

Turner, J. and Dankoski, M. (2008) Objective Structured Clinical Exams: A Critical Review. In Family Medicine, 40(8):574-8. 